\title{
Analyzing the Effects of PA Variations on the Performance of Phased Array Digital Predistortion
}

\author{
Nuutti Tervo, Marko E. Leinonen, Janne Aikio, Timo Rahkonen and Aarno Pärssinen \\ Faculty of Information Technology and Electrical Engineering (ITEE), University of Oulu, Finland
}

\begin{abstract}
This paper shows how digital predistortion of a phased array can benefit from the parametric variations over parallel power amplifiers (PAs). Different antenna configurations are simulated by varying the $P A$ input drive levels by the Monte-Carlo method. The error vector magnitude (EVM) at the steering angle and total radiated adjacent channel power ratio (TRACPR) are used as performance metrics. The simulation results indicate that array predistortion can benefit from the variations between the PAs to improve the EVM significantly. However, at the same time, the TRACPR performance is reduced. This gives a new trade-off to balance between in-band and outoff-band distortion in the fifth generation beamforming systems.
\end{abstract}

\section{INTRODUCTION}

The forthcoming fifth generation $(5 \mathrm{G})$ is suggested of occupying the millimeter wave frequency bands [1] Large antenna arrays are needed to compensate the high path losses as the physical dimensions of a single radiator drastically decreases. In order to achieve both high efficiency and high effective isotropic radiated power (EIRP), each antenna branch should be equipped with an individual power amplifier (PA) placed close to the antenna.

Traditionally, digital predistortion (DPD) has been used to enable PAs to operate close to the compression in order to maximize the efficiency. However, phased arrays challenge this concept as the PAs do not have individual baseband inputs. Furthermore, high-order modulation schemes may require linearization even for achieving decent error vector magnitude (EVM) [2]. Utilization of a single DPD for the complete array has been studied e.g. in [3]-[7]. However, the significance of large variations over parallel PA operation points have not been discussed in detail in the mentioned references. For example in [3], the effects of the parametric variations of individual PAs were limited to a fixed tapering scheme, which was used to reduce the sidelobes of the beam pattern. In this paper we highlight that any amplitude and phase variations can be utilized to enhance the EVM by allowing them to cancel each other in the desired direction. In other words, we could benefit from the component variations, amplitude errors of the phase shifters, the effects of mutual coupling [4], or any other differences in PA nonlinearities and utilize them for linearization. The required differences over the nonlinearities of individual RF branches can be also made in a controlled manner.

\section{NONLINEARITY IN BEAMFORMING ARRAYS}

\section{A. PA Array Figures of Merit}

If the waveform is assumed to experience identical nonlinearity in all parallel radio frequency (RF) branches, the beam of the nonlinear distortion is similar to the beam of the linear signal. [8] However, if the input amplitudes or the nonlinear characteristics of the individual PAs are varied, the beam pattern of the nonlinearity differs from the linear beam. Furthermore, in [9] it is discussed that also strong enough antenna coupling would cause differences on the nonlinearities of individual PAs. Hence, the nonlinearity of an array may depend on the direction of observation. This has been further discussed in [3] where the authors show that these differences can be actually utilized to compensate each other in the array far field. Hence, the standard figures of merit describing the nonlinear distortion of an array should be revised to have a right meaning from the system perspective [10].

As the root mean square (RMS) EVM describes the errors in the actual modulated data, it should be measured in the direction where the beam is directed. Out-off-band distortion is fundamentally interference to other users and systems that are operating at adjacent frequency bands. Hence, the array adjacent channel power (ACP) is a severe problem only if the system or user under interference is located in the direction of the radiated ACP. Even if the ACP pattern is directive, one should remember that an antenna array fundamentally only directs the power. Hence, if the array is linearized in one direction, the total radiated power (TRP) of the nonlinearity may still be conserved. Thus, a practical way to specify ACP is to integrate it over the PA elements, or equivalently integrate it over the three dimensional space. According to the third generation partnership project / new radio (3GPP/NR) [1], this is also considered to be the way of specifying the ACP in $5 \mathrm{G} \mathrm{mmW}$ systems.

TRP for the discrete set of directions can be calculated as

$$
\begin{aligned}
P_{\mathrm{TRP}} & =\frac{1}{2 N_{a z} N_{e l}} \sum_{n=0}^{N_{a z}-1} \sum_{m=0}^{N_{e l}-1}\left(\operatorname{EIRP}_{\phi}\left(\phi_{n}, \theta_{m}\right)\right. \\
& \left.+\operatorname{EIRP}_{\theta}\left(\phi_{n}, \theta_{m}\right)\right) \sin \phi_{n},
\end{aligned}
$$




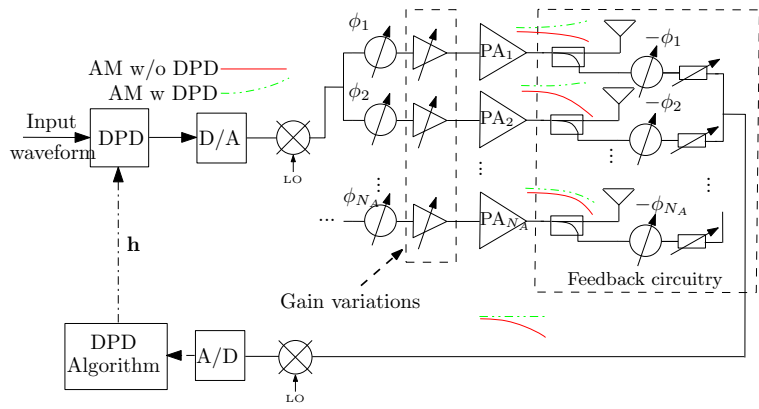

Fig. 1. Phased array DPD system with phased feedback.

where $\mathrm{EIRP}_{\phi}$ and $\mathrm{EIRP}_{\theta}$ denotes the EIRPs in $\phi$ and $\theta$ polarizations, $\phi$ and $\theta$ denotes the azimuth and elevation angles with discrete grid over $N_{a z}$ and $N_{e l}$ values, respectively. TRACPR can be calculated as a difference between total radiated ACP (TRACP) and total radiated channel power (TRCP) as

$$
\text { TRACPR }=\max \left\{\mathrm{TRACP}_{H}, \mathrm{TRACP}_{L}\right\}-\mathrm{TRCP},
$$

where the power is expressed in $\mathrm{dBm}$ and subindices $H$ and $L$ denotes the power of higher and lower adjacent channels. According to the best of the author's knowledge, TRACPR performance of a phased array with DPD has not been reported in the open literature.

\section{B. Digital Predistortion of a Phased Array}

Two main strategies has been proposed to linearize a phased array [3] with a single DPD. One can either try to linearize the individual PAs e.g. in least squares (LS) sense, or try to linearize the array response to a desired spatial direction. In this paper, we focus on the latter one. The block chart of the phased array utilizing single DPD is presented in Fig. 1. The phased feedback circuitry samples each PA output and applies a phase shift, which cancels the phase progression of the beam steering. Hence, the feedback mimics the behavior of the individual PA responses in the beamforming direction, i.e. it emulates the directive line-of-sight (LOS) radio channel. By using the combined feedback, standard DPD techniques can be used to linearize the whole array with single DPD. Other feedback methods proposed in the literature are single PA feedback [7], reference antenna [5] or timely-shared feedback [3], [6]. The channel-emulating phased feedback proposed in this paper enables fast DPD process and utilization of direction dependency of the nonlinear array response.

The nonlinear output characteristics of the individual PAs can be varied e.g. by controlling the gains of the corresponding RF branches. Thus, the nonlinear responses at each individual PA output, i.e. at individual antenna input can be expressed as [3]

$$
\begin{aligned}
z_{\text {out }, k}= & F_{A M}\left(w_{k} x\right) \\
& \exp \left(j\left(\arg \left(w_{k} x\right)+F_{P M}\left(w_{k} x\right)\right)\right),
\end{aligned}
$$

where $x$ denotes the input waveform, $w_{k}$ denotes the beamforming coefficient of $k$ th antenna, $F_{A M}$ denotes the amplitude to amplitude modulation (AMAM) and $F_{P M}$ the amplitude to phase modulation (AMPM) response of the PA, respectively. The free-space combining can be modelled by array factor for given antenna spacing and observation direction $(\phi, \theta)$. If all the antenna elements are identical, the theoretical nonlinear behavior in the array far field can be expressed as

$$
\begin{aligned}
& z_{A}(\phi, \theta)=\mathbf{F}_{A M}^{T}(\mathbf{w} x) \\
& \exp \left(j\left(\arg (\mathbf{w} x)+\mathbf{F}_{P M}(\mathbf{w} x)+\mathbf{k}^{T} \mathbf{r}\right)\right) F_{S E}(\phi, \theta),
\end{aligned}
$$

where $\mathbf{w}$ denotes the beamforming coefficients, $\mathbf{r}=\left[\mathbf{r}_{x}, \mathbf{r}_{y}, \mathbf{r}_{z}\right]^{T}$ includes the antenna element coordinates in cartesian coordinate system, $\mathbf{k}=\frac{2 \pi}{\lambda}[\sin (\theta) \cos (\phi), \sin (\theta) \sin (\phi), \cos (\theta)]$ denotes the three-dimensional wave vector, $F_{S E}(\phi, \theta)$ is the single antenna pattern, and $\lambda$ is the wavelength.

DPD of the complete array is modelled as

$$
y_{A_{\text {in }}}=\sum_{\substack{l=1 \\ l: \text { odd }}}^{N_{p}} h_{l}^{*}|x|^{l-1} x,
$$

where $h_{l}$ denotes the DPD coefficients and $N_{l}$ is the order of the polynomial. The coefficients for common array DPD are solved by minimizing the array response in the beam steering direction $\left(\phi_{d}, \theta_{d}\right)$ measured by the phased feedback circuitry. By using (4), minimisation of directive LS error can be written as

$$
\underset{\mathbf{h}, \phi=\phi_{d}, \theta=\theta_{d}}{\arg \min } \sum_{n=1}^{N_{n}}\left|\frac{1}{K_{\mathrm{A}}} z_{A}(n, \phi, \theta)-y_{A_{i n}}(n)\right|^{2},
$$

where $K_{\mathrm{A}}$ denotes the linear gain of the array in the desired direction, including both the power combining gain and beamforming gain, and $n$ denotes the sample index. When the PAs are driven with different levels, their nonlinear characteristics can vary significantly from each other. However, only the combined response is used to calculate the DPD coefficients. Hence, with DPD, the linearly amplifying PAs are expanding, which are actually linearizing the compression of the higher driven PAs [3]. This behavior is creating a notch for the distortion in the beamforming direction.

\section{Simulation Setup ANd Monte-Carlo ANALYSIS}

\section{A. PA Array Model and Simulation Parameters}

The simulation model in Matlab consists of PA model in time domain whereas the array and phase shifters are modelled in frequency domain. The transitions between the frequency and time domain models are calculated by using Discrete Fourier Transform (DFT). The PA model is a lookup table which is based on the AMAM \& AMPM measurements of the $13 \mathrm{GHz} 4$-stack $45 \mathrm{~nm}$ CMOS SOI PA [11]. The input waveform is $100 \mathrm{MHz}$ 


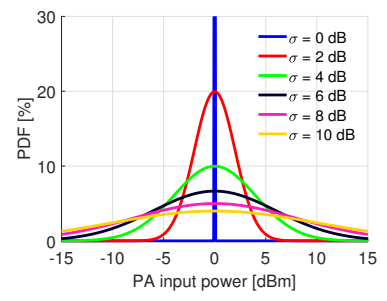

(a)

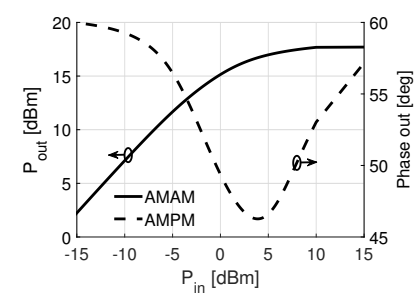

(b)
Fig. 2. (a) Distributions of PA input powers and (b) AMAM \& AMPM data of the used PA model.

wide, 64-QAM signal with raised cosine pulse shaping (roll-off 0.35). The antenna array is a uniform linear array (ULA) with varying number of identical $\lambda / 2$ spaced patch elements. The main lobe gain of each patch element is around $5 \mathrm{dBi}$. In the array model, impedance pulling of neighboring RF branches, or reflections from the antenna, both discussed e.g. in [4], are not taken into account.

As a DPD model, we use memoryless power series (5) with four coefficients that are solved by LS fitting between the combined feedback output and phased array input from (6). The simulations are performed over the azimuth plane with $1^{\circ}$ resolution. The input powers of the parallel PAs are assumed to follow normal distribution on a logarithmic scale with the mean of $0 \mathrm{dBm}$ and standard deviation of $\sigma_{s} \mathrm{~dB}$. The simulations are performed by varying $\sigma_{s}=\{0,2,4,6,8,10\}$ $\mathrm{dB}$ and the number of antennas in the ULA $\left(N_{a}=\right.$ $\{1,2,4,8,16,32,64,128,256\})$. The Monte-Carlo simulation is repeated 500 times for each $N_{A}$ and $\sigma_{s}$. The mapping of the PA input distributions to the used PA model is shown in Figs. 2a-b. The steering angle in the example was $0^{\circ}$, but the analysis holds for other steering angles as well.

The analysis is divided into three parts. In section III-B, we show the in-band distortion analysis for 32 element ULA as average beams and cumulative distribution functions (CDFs) of EVM over the Monte-Carlo tests. Section III-C conducts similar analysis for the ACP and in section III-D we extend the analysis explained in sections III-B and III-C for different number of antenna elements in ULA.

\section{B. Array In-band Distortion}

Average beam shapes of channel power and in-band distortion power (IDP) over the azimuth plane is shown in Fig. 3. The average is calculated over the MonteCarlo tests for 32 element ULA with different standard deviations of input powers. For the sake of clarity, only results with $\sigma_{s}=\{0,4,8\}$ are shown in the figure. The denoted beam patterns of the channel power corresponds the intended wideband beamshape. IDP is calculated by adding the EVM $[\mathrm{dB}]$ to the calculated channel power.

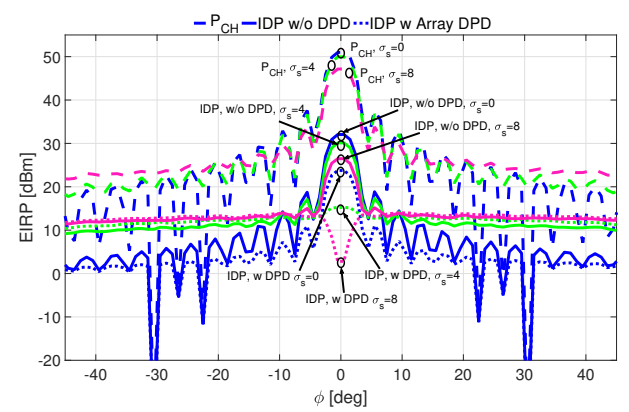

Fig. 3. Average beam shapes of channel power and IDP for 32-element ULA with and without DPD $\left(\sigma_{s}=\{0,4,8\}\right)$.

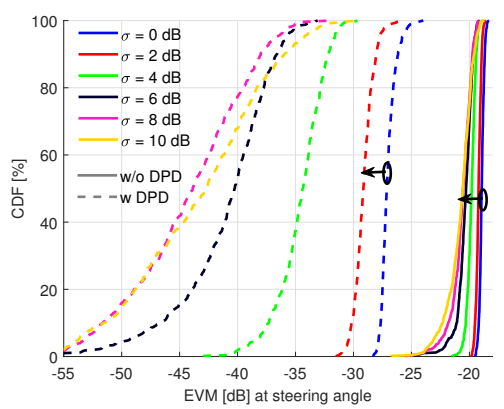

Fig. 4. CDFs of the simulated EVM in the steering angle for 32element ULA with and without DPD.

The directivity of the array is slightly decreasing as we increase the variations over individual PA elements. This can be seen as the compromized EIRP performance that is caused by the compression of the higher driven PAs. With $\sigma_{s}=0$, i.e. without variations, the beam shape of the IDP is similar compared with the channel power beam shape. However, as the variations are increased the IDP begins to create a notch for the beamforming direction, whereas it is increased in the other directions. Clearly, the nonlinearity depends now on the direction of observation and the beam is linearized only to the desired direction. This can be explained by the compressiveexpansive behavior of individual PAs as demonstrated in Fig. 1 [3].

CDFs of the EVM observed in the beamforming direction for 32-element ULA are given in Fig. 4. Without DPD, the EVM is increasing only a little bit as we increase the deviation of the input powers. When the PAs are driven with different levels, the nonlinearities of individual PAs become less correlated, which spreads the distortion power in the azimuth plane as it was observed from Fig. 3. With DPD, we can significantly boost this effect. More difference in the nonlinearities between the PAs we have, the better EVM can be potentially achieved in the array far field. For a single PA, such a small EVM would require large back-off or complex DPD model. By utilizing the array effect, the overall array response is smoother as the PAs are partly cancelling each other in 
the desired direction and the linearization can be focused on the combined response.

\section{Array Out-off-band Distortion}

Average beam shapes of channel power and ACP over the azimuth plane is shown in Fig. 5. Without variations, the beam shape of the ACP is similar compared with the channel power beam shape. When increasing the variations, the notch in the ACP is clearly observed. However, as the ACP specification was defined as TRP, the notch in the beamforming angle does not necessarily improve the TRACPR. Moreover, as the variations over parallel PAs are increases, the ACP spreads more in the azimuth plane. Hence, the created notch for the ACP in the desired direction slightly increase the ACP in other directions.

Fig. 6 shows the CDFs of TRACPR with and without DPD. Both channel power and TRACP is now integrated over the plane by using (1) and the TRACPR is the difference between those as presented in (2). Due to the fact that some of the PAs can potentially be driven even in saturation, the TRACPR increases as we increase the variations. Similar observation can be made for the TRACPR with DPD. The DPD utilizes the differences between the nonlinearities of the individual PA outputs to cancel each other in the beamforming direction. Hence, the expansion of the lower driven PAs makes some of the PAs more nonlinear, which potentially decreases the TRACPR performance. However, the DPD is still able to reduce TRACPR due to the fact that the distortion of the compressing PAs is dominating. The behavior of TRACPR is inverse compared with the behavior of the EVM when the variations between the PAs are increased.

\section{Increased Number of Nonlinear Array Elements}

The combined analysis on the effects of PA input power variations for larger arrays is collected to Fig 7. The figure shows the mean values of the EVM and TRACPR, over the Monte-Carlo tests, with and without DPD for different number of antennas and input drive level variations. Note that the subfigures are plotted on different scales to highlight the differences and the dynamics as a function of $\sigma_{s}$ and $N_{A}$. Without DPD, increasing the variations between the PAs slightly improves the EVM. However, the system needs enough antennas to benefit from the natural averaging effect. Increasing the number of antennas to be close to hundred also starts slightly decreasing the TRACPR. However, without DPD, the natural averaging effect on both EVM and ACPR is relatively small. Moreover, the DPD is actually amplifying the effect and hence it improves both EVM and TRACPR. The significant improvement in EVM is obtained by allowing the PA nonlinearities to cancel each other in the array far field. Furthermore, the DPD also improves the TRACPR, but the performance degrades as the PA variations are increased. However,

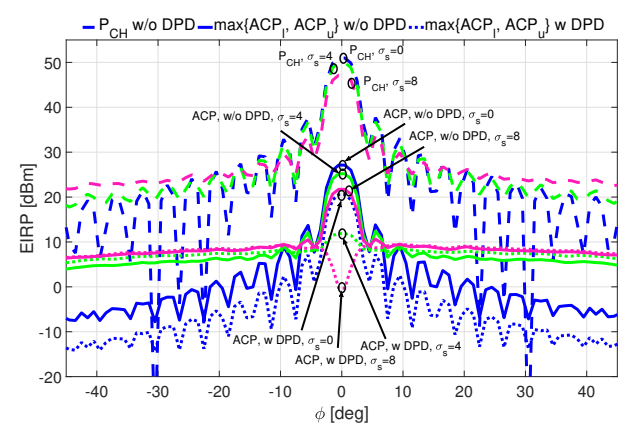

Fig. 5. Average beam shapes of channel power and ACP for 32element ULA with and without DPD $\left(\sigma_{s}=\{0,4,8\}\right)$.

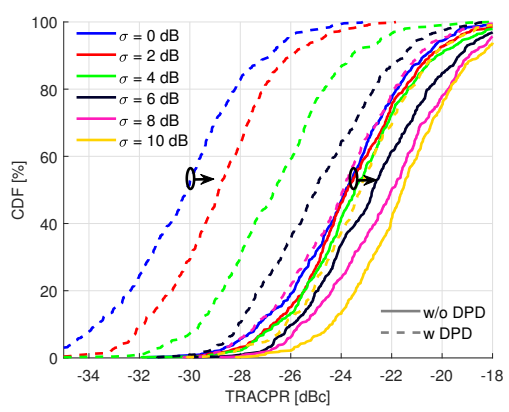

Fig. 6. CDFs of the simulated TRACPR for 32-element ULA with and without DPD.

with higher number of antennas, it is easier to benefit from the array DPD for both EVM and TRACP.

\section{CONCLUSION}

High data-rates require extremely good EVM performance, which sets tight requirements for the linearity of a phased array transmitter. We studied the EVM and total radiated ACP performance of phased array DPD by varying the PA input drive levels by the Monte-Carlo method. The simulations were performed with different number of antennas. Without DPD, minor improvement in the EVM was observed. However, with DPD the EVM was improving significantly as the variations and number of antennas were increased. Hence, the array DPD can benefit from the variations between the nonlinear characteristics of individual RF branches to improve the EVM close to the digital EVM limits. However, the TRACPR performance of the DPD was decreasing as we increased the variations between the PAs. This indicates that a practical linearization strategy of a phased array could improve TRACPR a certain amount while the EVM may be tuned according to the used high-order modulation scheme. In practice, the results indicates that the parallel PAs can be used to linearize each other in the beamforming direction and hence make EVM not dominated by the nonlinearity, but other nonlinearities such as phase noise of the local oscillator. The required differences over the nonlinearities of individual RF branches can be made 


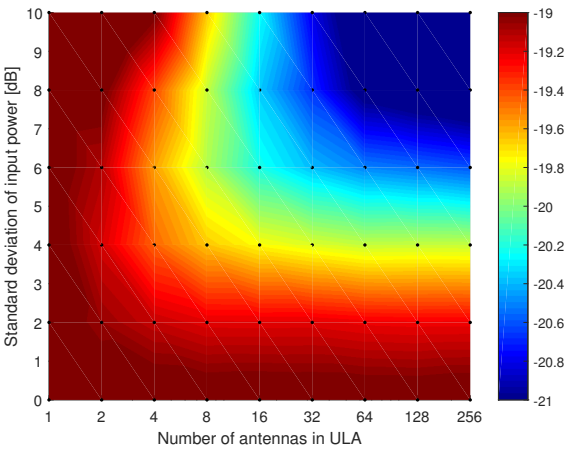

(a) EVM w/o DPD

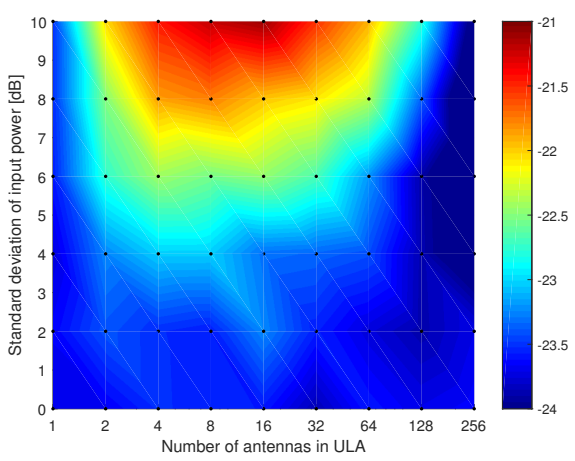

(c) TRACPR w/o DPD

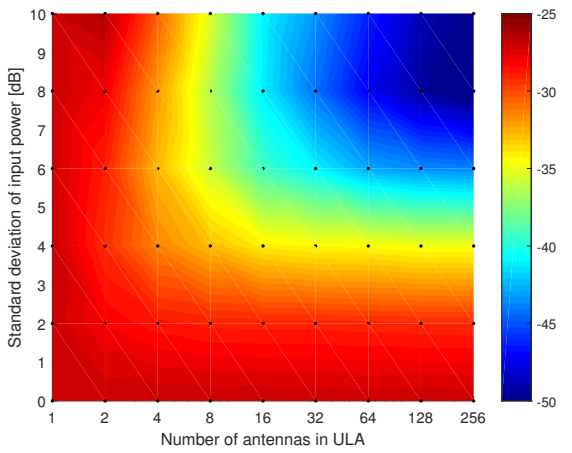

(b) EVM w DPD

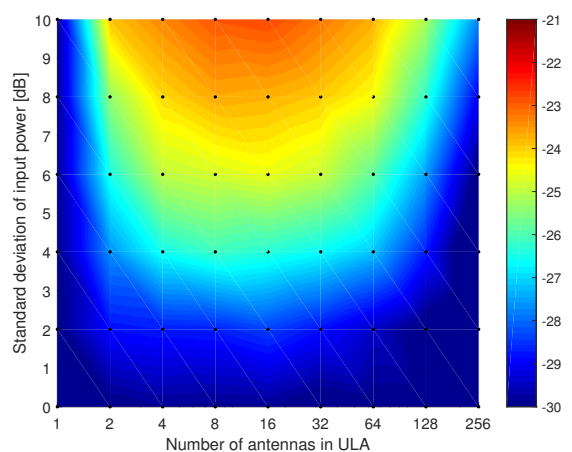

(d) TRACPR w DPD

Fig. 7. Simulated directive EVM and TRACPR results with different number of antennas and standard deviations of PA input powers. Note that the plots are on different scales purposely to highlight the dynamics with respect to $\sigma_{s}$ and $N_{A}$.

in a controlled manner e.g. by tuning the PA transistor biasing of individual RF branches and hence change the AMAM and AMPM behavior of individual PAs. By that we could significantly improve both linearity and efficiency of an array.

\section{ACKNOWLEDGMENT}

This research has been supported by Infotech Oulu Doctoral Programme, Finnish Funding Agency for Technology and Innovation (Tekes), Nokia Oyj, Esju Oy and CoreHW Oy. Furthermore, the research has been financially supported by Academy of Finland 6Genesis Flagship (grant 318927).

\section{REFERENCES}

[1] "3rd Generation Partnership Project; Technical Specification Group Radio Access Network; NR; Base Station (BS) radio transmission and reception (Rel 15)." [Online]. Available: http://www.3gpp.org/DynaReport/38-series.htm

[2] T. Tuovinen, N. Tervo, and A. Pärssinen, "Analyzing 5G RF System Performance and Relation to Link Budget for Directive MIMO," IEEE Transactions on Antennas and Propagation, vol. 65, no. 12, pp. 6636-6645, Dec 2017.

[3] N. Tervo, J. Aikio, T. Tuovinen, T. Rahkonen, and A. Pärssinen, "Digital predistortion of amplitude varying phased array utilising over-the-air combining," in 2017 IEEE MTT-S International Microwave Symposium (IMS), June 2017, pp. 1165-1168.
[4] C. Fager, K. Hausmair, K. Buisman, K. Andersson, E. Sienkiewicz, and D. Gustafsson, "Analysis of nonlinear distortion in phased array transmitters," in 2017 Integrated Nonlinear Microwave and Millimetre-wave Circuits Workshop (INMMiC), April 2017, pp. 1-4.

[5] Q. Luo, C. Yu, and X. W. Zhu, "Digital predistortion of phased array transmitters with multi-channel time delay," in 2018 IEEE Topical Conference on RF/Microwave Power Amplifiers for Radio and Wireless Applications (PAWR), Jan 2018, pp. 54-57.

[6] S. Lee, M. Kim, Y. Sirl, E. R. Jeong, S. Hong, S. Kim, and Y. H. Lee, "Digital predistortion for power amplifiers in hybrid mimo systems with antenna subarrays," in 2015 IEEE 81st Vehicular Technology Conference (VTC Spring), May 2015, pp. 1-5.

[7] L. Liu, W. Chen, L. Ma, and H. Sun, "Single-pa-feedback digital predistortion for beamforming mimo transmitter," in 2016 IEEE International Conference on Microwave and Millimeter Wave Technology (ICMMT), vol. 2, June 2016, pp. 573-575.

[8] E. G. Larsson and L. V. der Perre, "Out-of-band radiation from antenna arrays clarified," IEEE Wireless Communications Letters, 2018.

[9] K. Hausmair, S. Gustafsson, C. Snchez-Prez, P. N. Landin, U. Gustavsson, T. Eriksson, and C. Fager, "Prediction of Nonlinear Distortion in Wideband Active Antenna Arrays," IEEE Transactions on Microwave Theory and Techniques, vol. 65, no. 11, pp. 4550-4563, Nov 2017.

[10] M. Leinonen, N. Tervo, O. Kursu, and A. Pärssinen, "Out-ofBand Interference in 5G mmW Multi-Antenna Transceivers: Coexistence Scenarios," in European Conference on Networks and Communications (EuCNC), June 2018, pp. 1-5.

[11] J. Aikio, A. Sethi, R. A. Shaheen, R. Akbar, T. Rahkonen, and A. Pärssinen, "A Fully Integrated $13 \mathrm{GHz}$ CMOS SOI stacked Power Amplifier for 5G Wireless Systems," in 2017 IEEE Nordic Circuits and Systems Conference (NORCAS), 2017, pp. 1-4. 